\title{
Technological Features of Production Calcium- Alginate Microcapsules for Self-Healing Asphalt
}

\author{
Sergey Inozemtcev* and Evgeniy Korolev \\ Moscow State University of Civil Engineering, Yaroslavskoe shosse, 26, Moscow, 129337, Russia
}

\begin{abstract}
The effect of the formulation factors of calcium-alginate suspension preparation on the properties of calcium-alginate microcapsules was studied. The maximum useful volume of the reducing agent is set it was established, up to $93.8 \%$ of the total volume, in microcapsules, from the beating, to retain $2.31 \%$ sodium alginate and $10.5 \%$ reducing agent. The proposed mechanism for the formation of calcium-alginate capsules, which occurs due to shrinkage during the drying of a single drop of suspension, breaking the emulsion and forming an integral shell with a nucleus from the reducing agent.
\end{abstract}

\section{Introduction}

The length of highways with asphalt-concrete cover is about 15 million kilometres around the world, of them in the European Union 5 million kilometres, the United States - 8 million kilometres [1], and in Russia - 1 million kilometres [2]. The large network of roads requires significant financial costs for maintenance. Increase in the cost of restoring road structures occurs when untimely repairs. The economic development will suffer losses from the disruption of the logistics network of trucking and passenger traffic.

Traditional highways are multilayered structures in which various types of asphalt concrete are used for the upper layers. The projected service life of roads is 15 to 24 years [3]. However, intensive road traffic, difficult climatic conditions and poor road maintenance reduce the service life in Russia to $4 \ldots 8$ years [4]. The solution of the problem of increasing the lifespan of asphalt concrete in the pavement is the main task of materials science in the field of road construction.

The solution of the task is possible with the use of complex measures to modify the asphalt-concrete mixture. A promising direction is the creation of "smart" materials that are able to react independently to the change in structure (the formation of defects) and take measures to eliminate them during operation. The ability of the material to restore its own functionality in the structure, provided for its purpose, is usually called "self-healing" [5].

For example in building materials technology, self-healing is used both in polymeric materials and concretes on hydraulic or organic binders [6 ... 17]. Obviously, for various types of building materials, the mechanism of self-healing is different. In concrete, selfhealing technology with bacteria is used, which, in the process of life, produce calcium

* Corresponding author: inozemcevss@mgsu.ru 
carbonate, which fills the resulting fracture [6]. For polymeric materials, capsules with a reducing agent are used, which when the capsule is destroyed, reacts with the external environment and repairs the cracks [7].

There are difficulties for using similar technologies in asphalt concrete. Bacteria will die at temperatures of preparation of asphalt-concrete mixture from 140 to $185{ }^{\circ} \mathrm{C}$. Capsules with a reducing agent must be heat-resistant and withstand the load when compacting of the asphalt-concrete mixture.

Various technologies are used to produce microcapsules for asphalt concrete with a reducing agent. The technologies differ initial components, complexity of technological process and properties of capsules.

In work [12] the technology of two-stage coacervation for manufacturing microcapsules based on methanol-melamine-formaldehyde prepolymer modified with methanol is used.

In work [13] the technology of obtaining capsule shell, for emulsified reducing agent in water, from cellulose and polymer was proposed.

The authors of [14] proposed a three-stage method for obtaining microcapsules. In the first stage, styrene-maleic anhydride is stirred for 2 hours with water heated to $50{ }^{\circ} \mathrm{C}$. And then the $\mathrm{pH}$ of the solution is adjusted to 10 by $\mathrm{NaOH}$ and a reducing agent is introduced. In a second step, methanol-melamine-formaldehyde is added to the resulting emulsion and heated to $80{ }^{\circ} \mathrm{C}$ at a rate of $2{ }^{\circ} \mathrm{C}$ per minute. At the final stage, for the polymerization of the shell, the emulsion is aged for 2 hours at $80{ }^{\circ} \mathrm{C}$, after which the capsules obtained are filtered and dried in a vacuum oven.

A multi-step encapsulation technology in which water emulsified reducing agent is coated with a coating of urea and formaldehyde is proposed in [15].

The most technologically simple method of obtaining microcapsules was proposed by the authors [16]. At a constant mixing rate, sodium alginate and a reducing agent are successively added to the water. The resulting suspension is divided into individual capsules by means of a dropping funnel through a solution of calcium chloride. After filtration, the capsules are dried at a temperature of $40{ }^{\circ} \mathrm{C}$.

The authors of all the works reviewed use organic oils of different molecular weight as a reducing agent. However, the mechanism of action of such a reducing agent in asphalt concrete is the dissolution of aged bitumen components in it and a local decrease in brittleness. The result of the self-healing process is the restoration of the integrity of the structure of physic-chemical bonds in the composite by means of: wetting the surfaces of cracks, diffusion of molecules between surfaces and arbitrary scattering of molecules providing hardening [17]. In bitumen, self-healing occurs at the molecular level due to reversible hydrogen bonding with the formation of new cross-links and chains $[18,19]$ through ditopic and tritopic molecules [20].

In the case of oil-based oil rejuvenators, these processes do not occur, therefore, the effect of combining bitumen molecules is not observed. There is only a partial dilution of the components of bitumen in the rejuvenator, which contributes to reducing the potential for the development of a crack.

However, encapsulation is a promising technology in this direction, the development of which is possible when searching for the necessary reducing agent that provides molecular cross-linking in bitumen.

For a basis it is expedient to take a simple procedure of encapsulation with a low susceptibility to the properties of various reducing agents and not a large number of technological factors affecting the basic properties of the capsule shell. Such technology is the preparation of calcium-alginate microcapsules [16]. The purpose of this work is to establish the main technological factors that affect the properties of calcium-alginate microcapsules. 


\section{Materials and Methods}

For the production of calcium-alginate capsules, sodium alginate $\left(\mathrm{C}_{6} \mathrm{H}_{7} \mathrm{O}_{6} \mathrm{Na}\right)$ was used, which is the sodium salt of alginic acid extracted from brown algae. As a reducing agent used sunflower oil to exclude the effects of the features of the reducing agent. Sodium alginate was added to the water in various proportions and stirred for 2 minutes at a rotational speed of the drive of at least $2000 \mathrm{rpm}$ using an overhead stirrer [16]. The reducing agent was added to the resulting suspension and stirred for 2 minutes. The resulting emulsion was divided into capsules through a dropping funnel with an outlet opening of $0.71 \mathrm{~cm}$. The division was carried out in an aqueous solution of calcium chloride, prepared in advance in the proportions $\mathrm{CaCl}_{2} / \mathrm{H}_{2} \mathrm{O}=1 / 50$. After filtration and washing, the resulting capsules were dried in a furnace at a temperature of $40{ }^{\circ} \mathrm{C}$ for 12 hours.

Two-factor composite experimental design was implemented. Parameters of grinding $\left(X_{1}-\right.$ mass fraction of sodium alginate, $\% ; X_{2}$ - mass fraction of the reducing agent, $\%$ ) are selected as predictors. It was also proved during the research that polynomial regression model

$$
Y=B_{0}+B_{1} X_{1}+B_{2} X_{2}+B_{12} X_{1} X_{2}+B_{11} X_{1}^{2}+B_{22} X_{2}^{2}
$$

is suitable (hypothesis test for adequacy by means of $\mathrm{F}$-criterion) as a description. The basic levels and intervals of variation of the planning matrix are presented in Table 1.

Table 1. Zero levels and half-variations of the planning matrix.

\begin{tabular}{|l|c|c|}
\hline \multirow{2}{*}{ Parameters } & \multicolumn{2}{|c|}{ Factors of variation } \\
\cline { 2 - 3 } & $\boldsymbol{X}_{\mathbf{1}} \mathbf{\%} \%$ & $\boldsymbol{X}_{\mathbf{2}} \mathbf{\%}$ \\
\hline Zero level & 7.5 & 30 \\
\hline Half-variation & 2.5 & 10 \\
\hline
\end{tabular}

According to the plan, the dynamic viscosity $\eta$, the surface tension $\sigma$, the capsule diameter $d_{a}$, the capsule wall thickness $h_{k}$, the relative weight change $m_{\Delta}$ and the relative change in the diameter $d_{\Delta}$ of the capsules after drying were determined for each variant of the suspensions.

The dynamic viscosity of the suspension was determined using a rotational viscometer MCR 101, Anton Paar (Austria) with coaxial cylinders measure system. The dynamic viscosity was determined at a constant shear rate of $36 \mathrm{~s}^{-1}$ and a temperature of $25^{\circ} \mathrm{C}$.

The surface tension was determined on a KRUSS processor tensiometer (Germany) by the Wilhelm plate method. The surface tension was calculated on the basis of the measured force $\mathrm{F}$, the length of the wetted surface $\mathrm{L}$ and the wetting contact angle $\theta$ :

$$
\sigma=\frac{F}{L \cdot \cos \theta}
$$

Surface / interfacial tension measurements were carried out in the range $5 \ldots 100 \mathrm{mN} / \mathrm{m}$.

The loss of mass was determined by the ratio of the weight of the sample of the prepared capsules before and after drying. The diameter of the capsules was determined from the control measurements by means of a calliper with accuracy of $0.1 \mathrm{~mm}$.

The diameter was taken as the arithmetic average of the measured diameters of twenty capsules, selected randomly from the batch.

The thickness of the wall of the capsule was determined with a micrometer with an accuracy of $\pm 10 \mu \mathrm{m}$. The thickness of the wall of the capsule was taken as half the height of the compressed "tablet" obtained from the capsule. 
Mathematical planning and processing of experimental statistical models were carried out by the Gradient software complex [21].

\section{Results and discussion}

To obtain capsules with specified properties with a reducing agent, it is necessary to establish the limits of the variation of prescription factors. Sodium alginate is characterized by low water wettability. Particles of alginate powder tend to be highly aggregated in water to form agglomerates. Sodium alginate with water forms a colloidal solution with vigorous stirring. The size and shape of the capsules depends on the viscosity and surface tension of the resulting slurry, which varies on the content of sodium alginate and the reducing agent. The results of determining the dynamic viscosity and surface tension are presented in Table 2.

Table 2. Basic properties of suspensions of sodium alginate.

\begin{tabular}{|l|c|c|c|c|c|c|c|c|c|}
\hline \multirow{3}{*}{ Parameters } & \multicolumn{8}{|c|}{ The value of property indicators in accordance } \\
& \multicolumn{10}{|c}{ with the planning matrix } \\
\cline { 2 - 11 } & $\mathbf{1}$ & $\mathbf{2}$ & $\mathbf{3}$ & $\mathbf{4}$ & $\mathbf{5}$ & $\mathbf{6}$ & $\mathbf{7}$ & $\mathbf{8}$ & $\mathbf{9}$ \\
\hline$X_{1}, \%$ & 1.67 & 3.33 & 1.67 & 3.33 & 1.32 & 3.68 & 2.50 & 2.50 & 2.50 \\
\hline$X_{2}, \%$ & 6.67 & 6.67 & 13.33 & 13.33 & 10.00 & 10.00 & 5.29 & 14.71 & 10.00 \\
\hline$\eta, \mathrm{Pa} \cdot \mathrm{s}$ & 1.9 & 9.4 & 1.9 & 6.2 & 1.0 & 11.3 & 4.8 & 5.1 & 5.0 \\
\hline$\sigma, \mathrm{mN} / \mathrm{m}$ & 39.8 & 28.1 & 42.2 & 30.0 & 48.2 & 30.1 & 32.2 & 40.7 & 40.8 \\
\hline
\end{tabular}

In the next stage of preparation of capsules a suspension of sodium alginate is added dropwise to a solution of calcium chloride, where the process of gelling of alginate occurs, which is possible at a $\mathrm{pH}$ below 4 . Calcium ions bind ribbons of alginate macromolecules filling their pores and cavities $0.19 \ldots 0.20 \mathrm{~nm}$ in size, corresponding to the diameter of the $\mathrm{Ca}^{+2}$ ion. The reagent product is alginate gel. Intensive gelling occurs when pores are filled with calcium ions, providing cross-linking of macromolecules through substituted $\mathrm{Na}^{+}$ cations (Fig. 1).
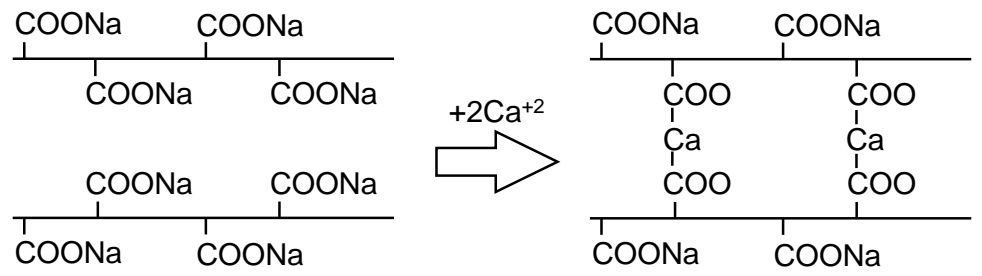

Fig. 1. Reaction of cross-linking of sodium alginate by calcium ions [22].

Then, the separated drops of alginate gel with the emulsified reducing agent are washed with distilled water and dried (Figure 2). 


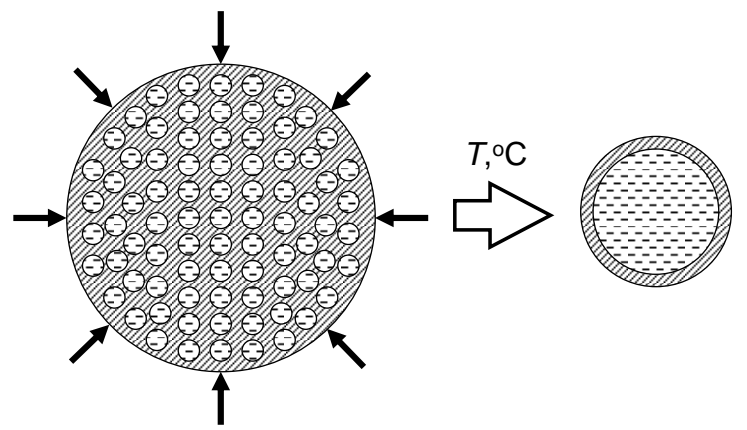

Fig. 2. Capsule formation during drying.

During drying, the excess water evaporates from the gel, the volume of the drop decreases, the emulsion inside the drop is destroyed and a calcium alginate capsule with a reducing agent is formed.

The results of determining the properties of the capsules are shown in Table 3.

Table 3. Properties of alginate capsules before and after drying.

\begin{tabular}{|l|c|c|c|c|c|c|c|c|c|}
\hline \multirow{2}{*}{ Parameters } & \multicolumn{8}{|c|}{ The value of property indicators in accordance } \\
& \multicolumn{1}{|c|}{ with the planning matrix } \\
\cline { 2 - 11 } & $\mathbf{1}$ & $\mathbf{2}$ & $\mathbf{3}$ & $\mathbf{4}$ & $\mathbf{5}$ & $\mathbf{6}$ & $\mathbf{7}$ & $\mathbf{8}$ & $\mathbf{9}$ \\
\hline$X_{1}, \%$ & 1.67 & 3.33 & 1.67 & 3.33 & 1.32 & 3.68 & 2.50 & 2.50 & 2.50 \\
\hline$X_{2}, \%$ & 6.67 & 6.67 & 13.33 & 13.33 & 10.00 & 10.00 & 5.29 & 14.71 & 10.00 \\
\hline$m_{\Delta}, \%$ & 7.14 & 5.10 & 4.18 & 3.81 & 4.64 & 4.08 & 7.20 & 3.86 & 4.06 \\
\hline$d_{a}, \mathrm{~mm}$ & 4.40 & 3.4 & 4.2 & 3.4 & 4.07 & 3.6 & 3.6 & 3.8 & 3.7 \\
\hline$d_{a}, \mathrm{~mm}$ & 2.48 & 2.41 & 2.84 & 2.76 & 2.23 & 2.21 & 2.29 & 2.88 & 2.68 \\
\hline$d_{\Delta}, \%$ & 1.78 & 1.41 & 1.48 & 1.23 & 1.82 & 1.63 & 1.57 & 1.32 & 1.38 \\
\hline
\end{tabular}

Note: $d_{a}{ }^{\prime}$ - average capsule diameters to dry; $d_{a}$ - average capsule diameters after drying; $d_{\Delta}$ - relative change in diameter of capsules after drying; $m_{\Delta}$ - relative change in weight of capsules after drying.

Parameters of the regression model were obtained based on the results of testing of alginate suspensions and calcium-alginate capsules (Table 4). The graphics interpretation of the regression model is reflected in figures 3 and 4 .

a)

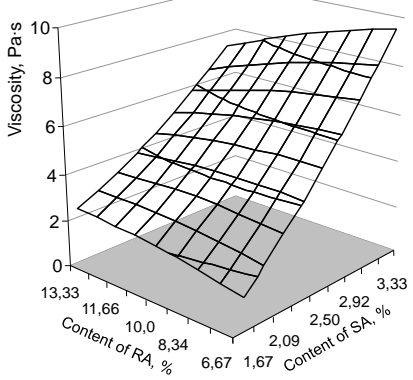

b)

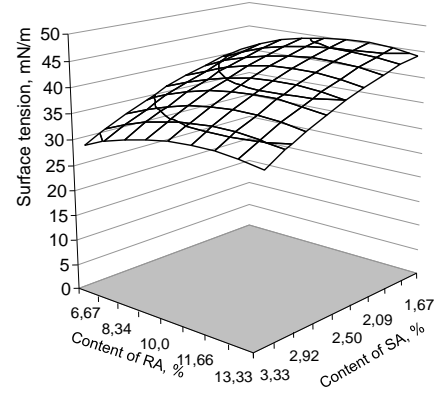

c)

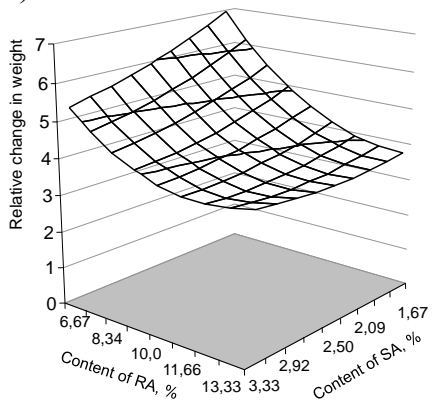

Fig. 3. Change in the dynamic viscosity (a) and surface tension (b) of the alginate suspension, the relative change in weight (c) of calcium-alginate particles from the content of sodium alginate (SA) and reducing agent (RA). 
Table 4. Parameters of statistical models.

\begin{tabular}{|c|c|c|c|c|c|c|c|}
\hline \multirow{2}{*}{ Parameters } & \multicolumn{7}{|c|}{ Parameters of regression model } \\
\cline { 2 - 8 } & $\boldsymbol{\eta}, \mathbf{P a} \cdot \mathbf{s}$ & $\boldsymbol{\sigma}, \mathbf{m N} / \mathbf{m}$ & $\boldsymbol{d}_{\Delta}, \mathbf{\%}$ & $\boldsymbol{m}_{\Delta}, \mathbf{\%}$ & $\boldsymbol{d}_{\boldsymbol{a}}, \mathbf{m m}$ & $\boldsymbol{h}_{\boldsymbol{c}}, \boldsymbol{\mu m}$ & $\boldsymbol{V}_{\boldsymbol{R} \boldsymbol{A}} / \boldsymbol{V}_{\boldsymbol{c}}, \boldsymbol{\%}$ \\
\hline$B_{0}$ & 4.99 & 40.84 & 1.61 & 4.06 & 2.68 & 30.70 & 93.3 \\
\hline$B_{1}$ & 3.30 & -6.17 & -0.02 & -0.40 & -0.02 & 14.81 & -3.28 \\
\hline$B_{2}$ & -0.35 & 2.03 & -0.14 & -1.12 & 0.19 & -4.19 & 1.87 \\
\hline$B_{12}$ & -0.81 & -0.14 & 0.005 & 0.42 & -0.0025 & -12.90 & 2.78 \\
\hline$B_{11}$ & 0.39 & -1.54 & -0.03 & 0.18 & -0.18 & 25.14 & -6.18 \\
\hline$B_{22}$ & -0.2 & -2.88 & 0.03 & 0.76 & 0.01 & 19.52 & -4.03 \\
\hline
\end{tabular}

Analysis of the results shows that the administration of sodium alginate increases the viscosity of the system, and an increase in the content of the reducing agent has a slight plasticization: the ratio of the coefficients $\left|B_{1} / B_{2}\right| \sim 10$, and the value of $B_{2}<0$ (Table 4). This is due to the increase in the number of sodium alginate macromolecules, which are randomly oriented in space and interacting with each other, contribute to the manifestation of non-Newtonian fluid properties. Sodium alginate is a surfactant in water, which leads to a decrease in surface tension with increasing content: coefficient $B_{1}<0$. The reducing agent does not affect the surface tension: the ratio $\left|B_{1} / B_{2}\right| \sim 3$ (Table 4).

The relative change in weight and diameter of the capsules decreases after drying with an increase in the content of sodium alginate and the reducing agent. Thus, $m_{\Delta}$ varies significantly higher. Sodium alginate more significantly affects $m_{\Delta}$ : the ratio $B_{1}\left(m_{\Delta}\right) / B_{1}\left(d_{\Delta}\right) \approx 20$; the reducing agent also more significantly affects $m_{\Delta}$ : the ratio $B_{2}\left(m_{\Delta}\right) / B_{2}\left(d_{\Delta}\right) \approx 8$.

The rate of change of the parameters under consideration (the derivative of the equation) differs depending on the variable factors:

$$
\begin{gathered}
\frac{d d_{\Delta}}{d X_{1}}=-0,06 \cdot X_{1}-0,015 ; \\
\frac{d d_{\Delta}}{d X_{2}}=1,52 \cdot X_{2}-0,135 ; \\
\frac{d m_{\Delta}}{d X_{1}}=-0,06 \cdot X_{1}+0,02 ; \\
\frac{d m_{\Delta}}{d X_{2}}=1,52 \cdot X_{2}-0,7
\end{gathered}
$$

The rate of change in diameter $d_{\Delta}$ and mass $m_{\Delta}$ of alginate capsules during drying with a change in the content of sodium alginate $X_{1}$ is 25 times smaller than when the content of the reducing agent $X_{2}$ is changed.

The effectiveness of the capsules, in the first place, is determined by the useful volume of the reducing agent, which it is able to release for eliminating the emerging defects in asphalt concrete (Fig. 4, c).

a)

b) c) 

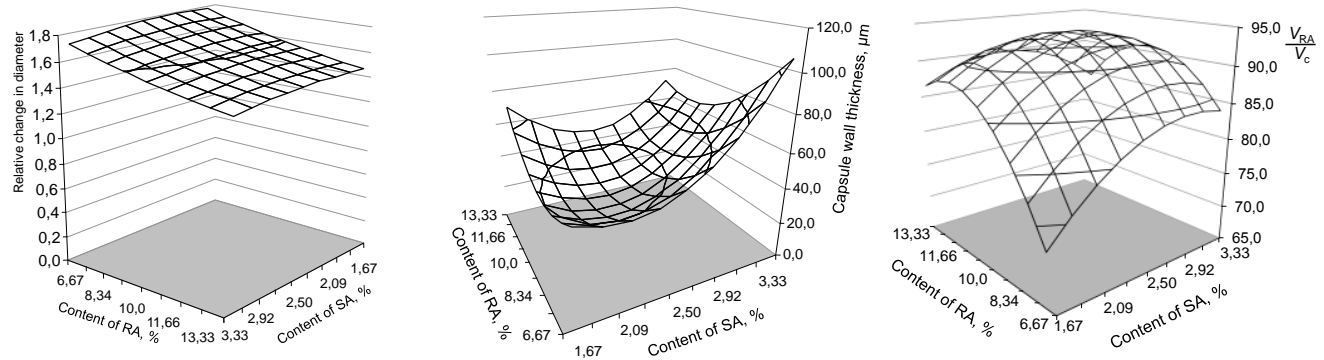

Fig. 4. Change in the average diameter of the capsules (a), the wall thickness of the capsules (b) and the ratio of the volume of the reducing agent to the volume of the capsule to $V_{R A} / V_{c}$ (c) from the content of sodium alginate (SA) and reducing agent (RA)

In accordance with the obtained experimental statistical model $V_{R A} / V_{\mathrm{c}}=f\left(X_{1}, X_{2}\right)$, the optimum formulation is: $X_{1}=2.31 \%, X_{2}=10.5 \%$. At the same time, $V_{R A} / V_{\mathrm{c}}==93.8 \%$.

\section{Conclusions}

1. The process of forming a capsule consists of shrinkage of individual droplets of the suspension during drying; destruction of the emulsion; formation of the shell with a nucleus from a reducing agent.

2. The shrinkage in the drying process decreases with an increase in the content of sodium alginate and the reducing agent. A more dense shell of alginate macromolecules forms and prevents the removal of excess moisture. Excess oil is an additional barrier.

3 . The microcapsules obtained from the slurry containing $2.31 \%$ sodium alginate and $10.5 \%$ reducing agent contain the maximum useful volume of the reducing agent, up to $93.8 \%$.

4. The resulting regression model is the basis for the manufacture of microcapsules containing reducing agents to restore the properties of bitumen in the composition of asphalt concrete.

This work was financially supported by the Ministry of Education and Science (state task \#7.6250.2017/8.9).

\section{References}

1. Environment at a Glance 2015: OECD Indicators. OECD Publishing. Paris. 104. (2015)

2. Russia in figures. 2017: Brief stat. / Rosstat - M. 511. (2017)

3. Decree of the Government of the Russian Federation No. 658 of May 30, 2017 on the standards of financial costs and the Rules for calculating the amount of budgetary allocations of the federal budget for capital repairs, maintenance and maintenance of highways of federal significance. 12. (2017)

4. Kamenchukov A.V. Evaluation of the reliability of non-rigid road clothes. The Fourth All-Russian Road Congress "Perspective technologies in the construction and operation of roads": Sat. sci. tr. - M.: MADI. 127-131. (2015)

5. Ghosh, Swapan Kumar. Self-healing materials: fundamentals, design Strategies, and applications (1st ed.). Weinheim: Wiley. VCH. 145. (2008) 
6. Jonkers HM, Schlangen E. A two components bacteria-based self-healing concrete. Concrete repair, rehabilitation and retrofitting II. Tailor \& Francis, London. 119-120. (2009)

7. Jin $\mathrm{H}$. et al. Self-healing thermoset using encapsulated epoxy-amine healing chemistry. Polymer. 53 (2). 581-587. (2012)

8. Boh B., Sumiga B. Microencapsulation technology and its applications in building // RMZ - Materials and Geoenvironment. 55. 3. 329-344. (2008)

9. Wilson G.O., Magnus H. Self-healing systems for industrial and marine protective coatings. Journal of Protective Coatings \& Linings. 28. 8. 18-21. (2008)

10. Kirkby E.L. et al. Embedded shape-memory alloy wires for improvements of selfhealing // Advanced Functional Materials. 18. 17. 2253-2260. (2008)

11. Martin D. Hager, Sybrand van der Zwaag, Ulrich S. Schubert Self-healing Materials / Springer. 413. (2016)

12. J.F. Su, E. Schlangen, J. Qiu Design and construction of microcapsules containing rejuvenator for asphalt // Powder technology 235, 563-571.

13. Raquel Casado Barrasaa, Víctor Blanco Lópeza, Carlos Martín-Portugués Montoliua, Verónica Contreras Ibáñezb, Josefina Pedrajasc, Julio Santarénd Addressing durability of asphalt concrete by self-healing mechanism // Procedia - Social and Behavioral Sciences. 162. 188 - 197. (2014)

14. J.F. Su, E. Schlangen. Synthesis and physicochemical properties of novel high compact microcapsules containing rejuvenator applied in asphalt // Chemical Engineering Journal. 198-199. 289-300. (2012)

15. Bin Xue, Huifeng Wang, Jianzhong Pei, Rui Li, Jiupeng Zhang, Zepeng Fan Study on self-healing microcapsule containing rejuvenator for asphalt // Construction and Building Materials. 135. 641-649. (2007)

16. T.Al-Mansooria, R.Micaeloabc, I.Artamendid, J.Norambuena-Contrerasae, A.Garcia. Microcapsules for self-healing of asphalt mixture without compromising mechanical performance // Construction and Building Materials. 155. 1091-1100. (2017)

17. Amir Tabaković, Erik Schlangen. Self-Healing Technology for Asphalt Pavements // Advances in Polymer Science book series. 273. 285-306. (2015)

18. Fisher H. Self repairing materials - dream or reality? // Natural Science. 2. 8. 873-901. (2010)

19. Qiu J. et al. Investigation of self healing capability of bituminous binders // Road Materials Pavement Design. 10. 1. 81-94. (2009)

20. Cordier P. et al. Self-healing and thermoreversible rubber from supramolecular assembly // Nature. 451. 977-980. (2008)

21. The program complex "Gradient" [Electronic resource] url: http // libv.org.

22. Koryachkina S.Ya., Prigarina OM Scientific foundations of food production: a textbook for higher professional education / Eagle: FGBOU HPE "State University of UNPK". 377. (2011) 